\title{
Quantum Harmonics for Quantum Engineering
}

\section{Budnik S*}

President, UTG-PRI TD., Tel Aviv, Russia

\begin{abstract}
In this article we offer to enhance the standard model of a bosonic superconducting cosmic string and model it in our quantum harmonic system to enable the creation of flexible (folded) quantum computers, I Phones and TVs, engineless quantum transmission and propelling devices for cars and aircrafts, super fluid propulsion, levitation and teleportation.
\end{abstract}

Keywords: Bosonic; Tunnelling; Cosmic; Fermionic; Teleportation; Quantum computers

\section{Elaboration}

Accordingly, and contrary to the common bosonic string model in Figure 1, we model our ultracold hollow cylindrical superstring (Figure 2) as a space time piercing string within the overlapping counterrotating magnetic fields. (Compare with the space time piercing ability of neutrinos and their left-right counter-spinning ability).

Our tunneling superstring system in Figure 2 consists of the open left entry to trap fermionic atoms in the vacuum vortex core, harmonizes them in vertex by shifting counter-rotating magnetic fields in a quasi Casimir effect to unify them in a superimposed magnetic field in quantum squeeze junction (Figure 3), and then superconducts them via superstring's right exit in mass propagation [1-3]. The system in Figure 3 functions similar to a musical squeezebox harmonika or accordion (see image below) which expands and contracts its bellows by using trapped air to create pressure and vacuum and produce musical sounds [4].

\section{Accordion}

Similarly to accordion functions, our quantum harmonic system

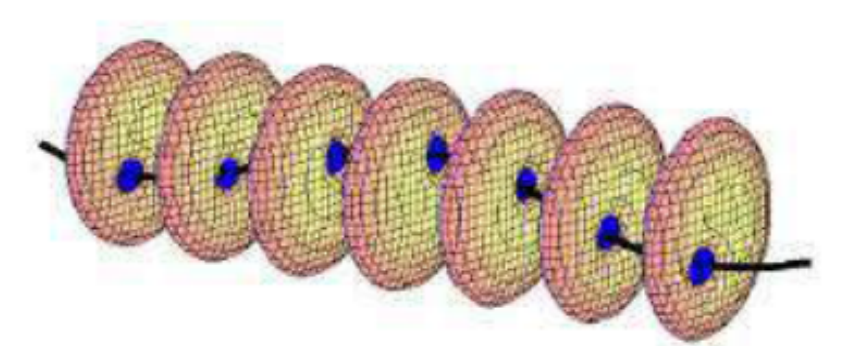

Figure 1: Super conducting Cosmic String.

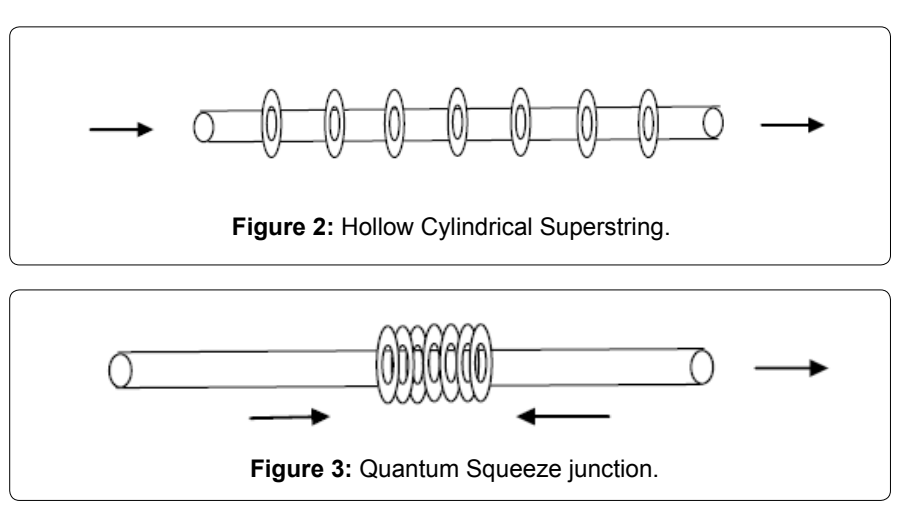

in Figure 3 shifts external magnetic fields back and forth over ultracold Majorana fermions trapped and compressed in the rotating tube of the superconducting superstring. In the lab, such a system can be represented by a vacuum tube with numerous counter-rotating electromagnets sliding back and forth over the tube and its trapped ultra cold particles. To make this system work as a cold fusion reactor, we would direct the particles beam from our quantum harmonic generator into the chamber with liquid helium and neon to interact there with solar neutrinos $[5,6]$. Our quantum model represents the classical and quantum motion of photons, etc. in a rotating string. The spin motion per Bargmann-Michel-Telegdi equation is considered in the rotation tube and rotating system in acceleration of charged particles. In fact, neutral particles photons, neutrons, etc. can be accelerated by rotating tube. The specific characteristics of the mechanical systems in the rotating framework follow from the differential equations describing the massive body in the noninertial systems.

Let be the Lagrange function of a point particle in the inertial system as follows:

$$
m=\frac{d_{v 0}}{d_{t}}=\frac{\partial U}{\partial r^{\prime}} L_{0}=\frac{m v_{0}^{2}}{2}-U
$$

With the following equation of motion

$$
m=\frac{d_{v 0}}{d_{t}}=\frac{\partial U}{\partial r^{\prime}}
$$

\section{Conclusion}

Because our ultra cold superstring is nonrelativistic, it is not constrained to the multidimensional spacetimes in which superstrings are usually studied in high-energy physics. It is the first harmonic condensed matter system proposed, where super conductivity in macroscopic quantum phenomena can be studied experimentally. Accordingly, our theory and model enable to create a super fluid propulsion system, quantum computers, IPhones and TVs, nanoturbines for cars, aircrafts and power stations, and quantum generator for portable cold fusion energ. In further application of our technology, new class of vehicles can be operated in levitation and superfluid propulsion, and energy teleported. Moreover, In

*Corresponding author: Solomon Budnik, President, UTG-PRI TD., Tel Aviv, Russia, E-mail: s.b0246@gmail.com

Received July 08, 2015; Accepted December 30, 2015; Published January 01 2016

Citation: Budnik S (2016) Quantum Harmonics for Quantum Engineering. J Electr Electron Syst 5: 170. doi:10.4172/2332-0796.1000170

Copyright: (c) 2016 Budnik S. This is an open-access article distributed under the terms of the Creative Commons Attribution License, which permits unrestricted use, distribution, and reproduction in any medium, provided the original author and source are credited. 
our quantum model, physical/molecular data of the object can be photonically compressed, tunneled via our quantum tube and then amplified/reassembled at given destination. The eternal question: why cosmic strings aren't detected by gravitational waves, is answered in assumption that in a quantum state, such mini strings never meet or spark, and function at zero point gravity, in anti-gravity or repelling gravity. Such cosmic mini strings create mini black holes, and hence cannot be detected by gravitational waves. When twin superstrings of matter create a macroscale black hole, as explained in our $11 \mathrm{pp}$. Theory of Unified Matter, we might detect them by gravitational waves.

\section{References}

1. Budnik S (2013) Theory of Transitional Universe (New Model of Space-Time) Social Science Research Network.
2. Kanai T, Minemoto S, Sakai H (2005) Quantum interference during high-order harmonic generation from aligned molecules International weekly journal of science 435: 470-474.

3. Bhattacharya M, Stoutimore MJA, Osborn KD, Mizel A (2012) Understanding the damping of a quantum harmonic oscillator coupled to a two-level system using analogies to classical friction. 1-7.

4. Chakeres DW, Vento R (2015) The Association of the Neutron, and the Quantum Properties of Hydrogen, with the Prime Numbers 2, 3, 5, 7, 11 Physics and Mathematics 6: 2145-2157.

5. Pigeon S, Fusco L, Xuereb A, Paternostro M (2015) Thermodynamics of trajectories of a quantum harmonic oscillator coupled to $N$ baths. Queens University press.

6. Paul PM, Toma ES, Breger P, Mullot G, Ague F, et al. (2001) Observation of a Train of Attosecond Pulses from High Harmonic Generation Science Magazine 292: 1689-1692. 